\title{
Dosimetric evaluation of internal shielding in a high dose rate skin applicator
}

\author{
Françoise Lliso, SD', Domingo Granero, PhD², Jose Perez-Calatayud, PhD',3 Vicente Carmona, SD', \\ M Carmen Pujades, MSc', Facundo Ballester, $\mathrm{PhD}^{4}$ \\ ISección de Radiofisica en Radioterapia, Hospital Universitario LA FE, Valencia, Spain, 2 Servicio de Radiofísica, ERESA, \\ Hospital General Universitario, Valencia, Spain, 3Radiotherapy Department, Clinica Benidorm (Alicante), Spain, \\ ${ }^{4}$ Departament de Física Atòmica, Molecular i Nuclear, Universitat de Valencia, València, Spain
}

\begin{abstract}
Purpose: The Valencia HDR applicators are accessories of the microSelectron HDR afterloading system (Nucletron) shaped as truncated cones. The base of the cone is either 2 or $3 \mathrm{~cm}$ diameter. They are intended to treat skin lesions, being the typical prescription depth $3 \mathrm{~mm}$. In patients with eyelid lesions, an internal shielding is very useful to reduce the dose to the ocular globe. The purpose of this work was to evaluate the dose enhancement from potential backscatter and electron contamination due to the shielding.

Material and methods: Two methods were used: a) Monte Carlo simulation, performed with the GEANT4 code, $2 \mathrm{~cm}$ Valencia applicator was placed on the surface of a water phantom in which $2 \mathrm{~mm}$ lead slab was located at $3 \mathrm{~mm}$ depth; b) radiochromic EBT films, used to verify the Monte Carlo results, positioning the films at 1.5, 3, 5 and $7 \mathrm{~mm}$ depth, inside the phantom. Two irradiations, with and without the lead shielding slab, were carried out.

Results: The Monte Carlo results showed that due to the backscatter component from the lead, the dose level raised to about $200 \%$ with a depth range of $0.5 \mathrm{~mm}$. Under the lead the dose level was enhanced to about $130 \%$ with a depth range of $1 \mathrm{~mm}$. Two millimeters of lead reduce the dose under the slab with about $60 \%$. These results agree with film measurements within uncertainties.

Conclusions: In conclusion, the use of $2 \mathrm{~mm}$ internal lead shielding in eyelid skin treatments with the Valencia applicators were evaluated using MC methods and EBT film dosimetry. The minimum bolus thickness that was needed above and below the shielding was $0.5 \mathrm{~mm}$ and $1 \mathrm{~mm}$ respectively, and the shielding reduced the absorbed dose delivered to the ocular globe by about $60 \%$.

Key words: skin brachytherapy, film dosimetry, Monte Carlo methods.

\section{Purpose}

The Valencia high dose rate (HDR) applicators, hereafter in the text "VLCAPP", are accessories of the microSelectron HDR afterloading system (Nucletron BV, Veenendaal, the Netherlands). The applicators are cup-shaped and tungsten shielded to limit the dose to the irradiation area and they are intended to treat surface lesions utilizing a contact treatment technique. The VLCAPP were designed on the base of the Leipzig applicators [1-4], from the same vendor, in order to overcome the principal dosimetric problems that these applicators exhibit, a very heterogeneous dose distribution in planes parallel to the skin surface. The dose distribution shows a significant penumbra reducing the useful irradiation field size of the applicators and the prescription at a depth on axis would not guarantee that the entire layer receives the intended dose.
There were six Leipzig applicators with inner diameters of 1,2 , and $3 \mathrm{~cm}$. Three of them used a source moving parallel to the treatment surface, $\mathrm{H} 1, \mathrm{H} 2$, and $\mathrm{H} 3$. The remaining three used a source which moved perpendicularly to the treatment surface, V1, V2, and V3. Two VLCAPP were developed and are commercially available with inner diameters of 2 and $3 \mathrm{~cm}$, named as Valencia applicators $\mathrm{VH} 2$ and $\mathrm{VH} 3$ respectively, where the source has a parallel orientation with respect to the treatment surface. The design details and an extensive dosimetric characterization were presented by Granero et al. [5]. Briefly, the applicators were equipped with a fixed flattening filter that provides a uniform dose distribution in planes parallel to the contact surface at a given depth along the cup axis. In a plane perpendicular to the axis of the applicators at a depth of $3 \mathrm{~mm}$, the dose heterogeneity was within $5 \%$. For more details about the isodose distributions the read- 
er is referred to the study of Granero et al. [5]. As described elsewhere [6-9] HDR brachytherapy with standardized surface applicators offers an effective alternative treatment technique to other radiation therapy modalities for the treatment of small superficial non melanoma skin cancers. The applicators can be used for the treatment of skin tumours, oral cavity, vaginal cuff, etc. When there is a critical structure located only at a few millimeters under the tumor, like for example in case of the eyelid, an internal shielding is very useful to reduce the dose to this structure. Dose perturbation at different material interfaces has been extensively studied for many years for different beam energies and modalities: electron beams [10], $\mathrm{kV}$ photon beams [11-13], and high-energy and gamma photon beams [14-16]. The perturbation depends on beam energy, field size, thickness, width, position, and atomic number of the heterogeneity creating the interface. It may be expected that when an internal lead shielding is used in the case of HDR Ir-192 treatment, apart from the attenuation a strong dose enhancement is observed in the tissue on the backscatter side adjacent to the lead slab and also in the forwardscatter side.

The aim of this work was to evaluate these potential effects when an internal shielding is used with the VLCAPP.

\section{Material and methods \\ Monte Carlo simulation}

The Monte Carlo (MC) simulation was performed with the GEANT4 code (Version 9.0) [17], to score absorbed dose. GEANT4 code, geometry of the VH2 and VH3 applicators and the complete process involved in analyzing the data were described in more detail in previous publications $[2,5]$. Specific details to this study are the following. Cylindrical rings $0.5 \mathrm{~mm}$ in thickness and $0.1 \mathrm{~mm}$ in depth were used to score absorbed dose. The $0.1 \mathrm{~mm}$ thickness of the scoring voxels was necessary due to high dose gradient present in this direction. The number of primary photon histories that were generated in each simulation is $3 \times 10^{8}$, using a cut off energy for photons and electrons of $1 \mathrm{keV}$. The VH2 applicator was chosen as it had an adequate size in relation to the size of the eyelid. Two simulations were done. The first one consisted in the applicator located on a cylindrical water phantom $10 \mathrm{~cm}$ in diameter and $10 \mathrm{~cm}$ in height without any heterogeneity. This phantom size was chosen as a representative size of typical applications. The second simulation was as the first one but with a lead heterogeneity of $2 \mathrm{~mm}$ thickness immersed in the water phantom at $3 \mathrm{~mm}$ depth i.e. at $3 \mathrm{~mm}$ from the window of the applicator.

\section{Radiochromic film dosimetry}

Gafchromic EBT dosimetry films (International Specialty Products) were used to verify the MC results in the arrangement showed in Fig. 1. A solid polystyrene slab phantom was used for the measurements. The film portions were positioned at four different depths: 1.5, 3, 5, and $7 \mathrm{~mm}$, sandwiched between the phantom slabs, parallel to the applicator surface. The thicknesses of the spacers were chosen in order to be representative of the penetration of the beam; one of the films was located at the prescription depth of $3 \mathrm{~mm}$. In order to perform a more straightforward comparison, two irradiations were carried out with the same setup except that in the first measurement, the films were stacked between the polystyrene slabs and in the other measurement, $2 \mathrm{~mm}$ thick polystyrene slab located at $3 \mathrm{~mm}$ depth was substituted by a lead shielding slab. A unique Gafchromic EBT sheet was used from which portions were cut into rectangular strips exceeding the applicator size for more than $1 \mathrm{~cm}$ in order to avoid the changes in the film due to the damage of the cut edges [18]. As it is seen in Fig. 1, the films were irradiated with the applicator on top of the phantom with a dwell time of the source calculated to deliver a dose to the film at $3 \mathrm{~mm}$ depth of about 2 Gy. A flatbed scanner (EPSON 10000XL) was used to scan the films following the recommendations found in a literature [19-21]. Briefly, each film was scanned prior to the irradiation and $24 \mathrm{~h}$ after the irradiation [22] in order to subtract the background [23]. The films were located at the centre of the scan bed in order to minimize the effect of the scanner heterogeneity, scanned 4 times and digitized in a consistent orientation. The images were acquired in the 48 bit colour red-green-blue (RGB) mode with the SilverFast LaCie software and saved as tagged image file format (TIFF), using a resolution of $100 \mathrm{dpi}$. The calibration of EBT films was performed at our department in Co-60 beam. Although a non negligible energy dependence of response has been recently reported [24] for EBT films irradiated with $\mathrm{X}$-ray beam qualities ranging between $10 \mathrm{kVp}$ and $15 \mathrm{MVp}$ and scanned with a flatbed scanner, from the results of that publication it is seen that

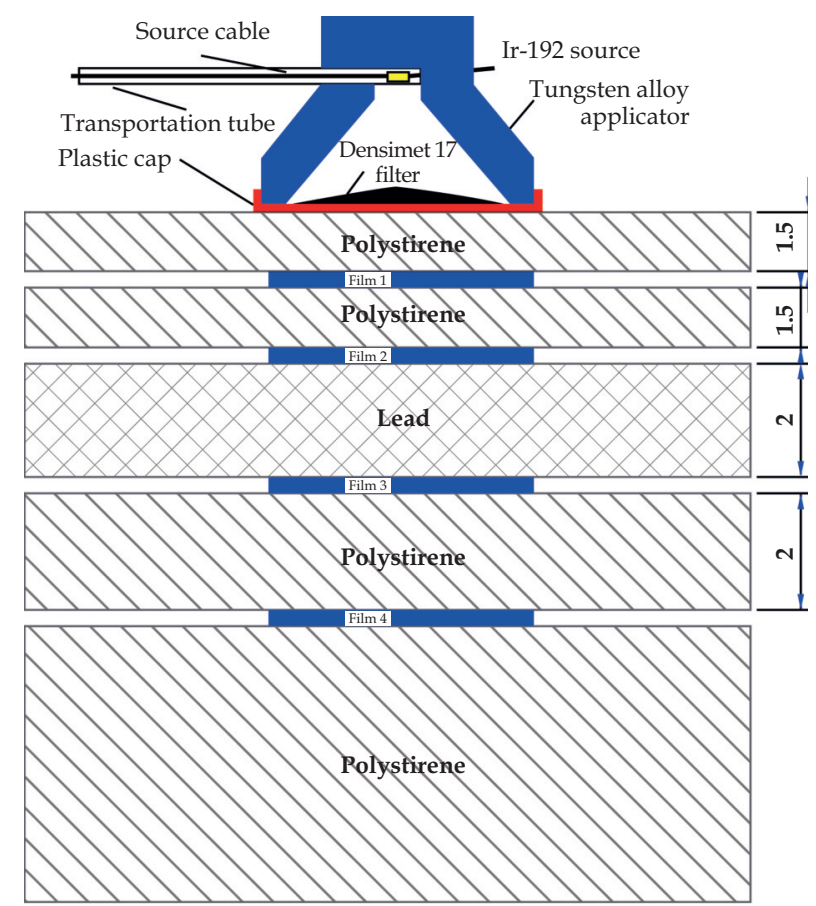

Fig. 1. Scheme of the measurement setup with the applicator on top of the phantom. Longitudinal section. Distances are indicated in $\mathrm{mm}$ only for clarification. The figure is not scaled 


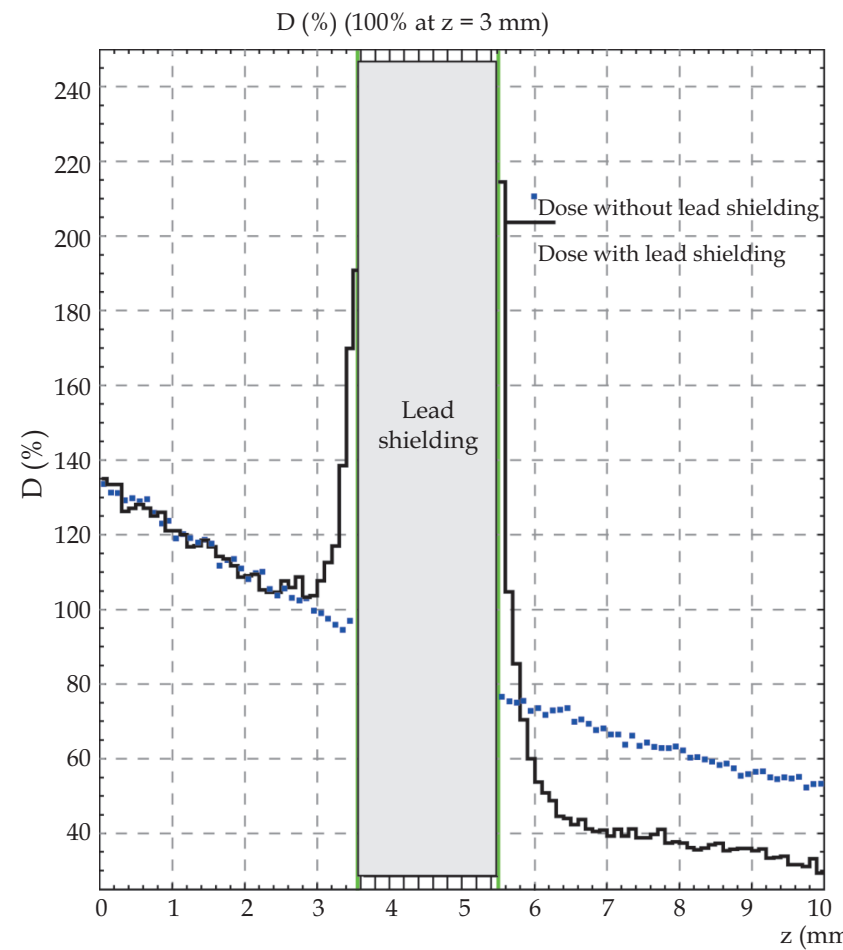

Fig. 2. Absorbed depth dose curves obtained with Monte Carlo. The dotted line is the depth dose curve corresponding to the water phantom and the solid line corresponds to the curve obtained with the lead slab inside

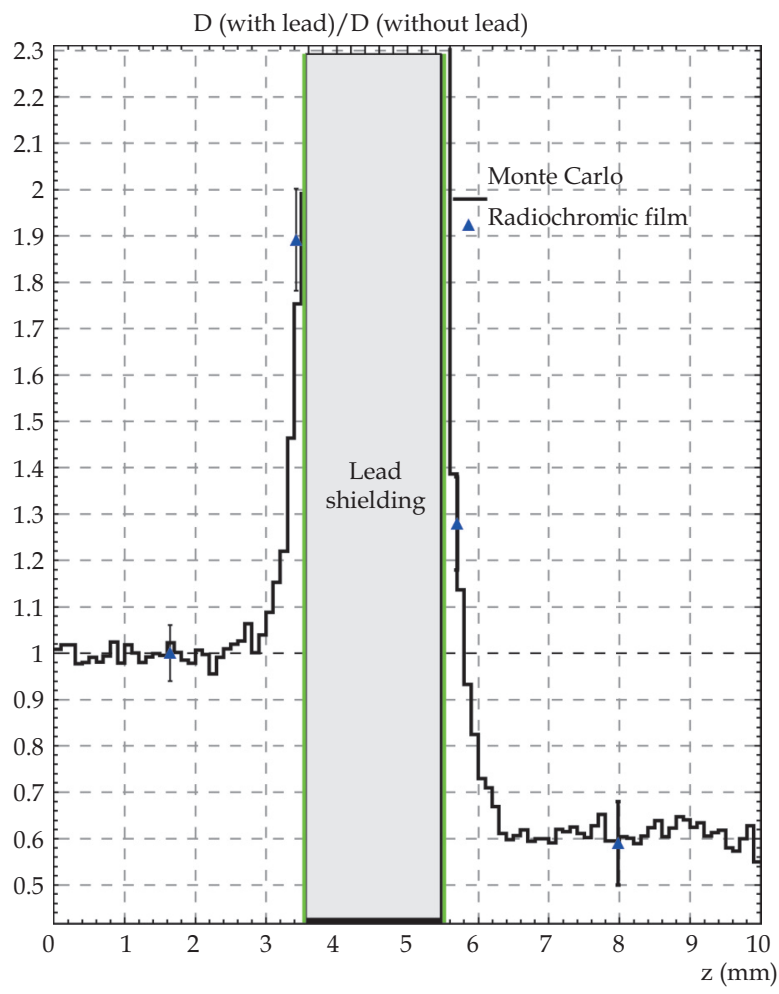

Fig. 3. The solid line corresponds to the ratio of the two depth dose curves obtained with Monte Carlo, with and without the lead shielding. Superimposed are the results obtained with radiochromic films indicated by triangles the variation in optical density (OD) between the Co-60 beam quality and the Ir-192 quality was very small. The MatLab 7.0 (Math works, Natick, MA) software was used to obtain the red channel information, to filter noise by means a $2 \mathrm{D}$ median filter of $5 \times 5$ pixels, and to obtain the net OD (by the subtraction of the background) and the corresponding absorbed dose.

\section{Results and discussion}

Figure 2 shows the results obtained from the MC simulation. When $2 \mathrm{~mm}$ lead shielding was in place, certain overdosage could be observed in the vicinity due to the backscattered component of the radiation, the dose level was raised to about $200 \%$ being the depth range of $0.5 \mathrm{~mm}$ perturbation. On the other hand the electron contamination under the lead produces an enhancement of the dose level to about $130 \%$ with a depth range of $1 \mathrm{~mm}$. The values of the range of the perturbation produced before and behind the lead shielding, providing the bolus thicknesses that had to be located besides the shielding: $0.5 \mathrm{~mm}$ before and $1 \mathrm{~mm}$ after the lead, respectively, were needed to attenuate the backscattered and forwarded radiation.

The ratio between the values obtained with both radiochromic measurements at $1.5,3,5$, and $7 \mathrm{~mm}$ were used to compare with the MC results. In Fig. 3 the results from the EBT film dosimetry are shown superimposed to the $\mathrm{MC}$ results. The uncertainty bars $(k=1)$ for these values (that are also shown) were calculated assuming an uncertainty for the absorbed dose values obtained with EBT films of 5\% [21]. The uncertainties in the MC simulations were less than $5 \%$ for both simulations, with and without the lead heterogeneity. From Fig. 3 it is also seen that the use of an internal $2 \mathrm{~mm}$ thick lead shielding, being the thickness value limited by the patient comfort, gives a dose reduction of about $60 \%$. In addition to that, a reduction of about $10 \%$ could be found in the clinical practice with the bolus in place. As it is seen from Fig. 3, the results obtained with film dosimetry agree within experimental uncertainties with the MC results. The MC simulation was performed according to the experimental setup that was limited by the polystyrene slabs availability and taking into account the thickness of the films, $0.234 \mathrm{~mm}$. This was the reason why the lead slab was not located at $3 \mathrm{~mm}$ depth but at $3.468 \mathrm{~mm}$ (see Figs. 2 and 3). Although the scope of our work was limited to the dosimetric issue of the type of applicator in cases where the user wants to apply a shield for underlying clinical structures, it proposes a solution to a problem that occurs in the clinical practice. This was the case of the backscattered radiation when an internal shielding was used in clinical electron beams in lips or eyelids treatments.

\section{Conclusions}

The use of an internal lead shielding in eyelid skin treatments with the Valencia applicators was evaluated using the MC methods and EBT film dosimetry. The results obtained from both methods agree within uncertainties. The minimum bolus thickness that must be added in order to remove the dose contamination, above and below the 
shielding, was calculated, resulting in values of $0.5 \mathrm{~mm}$ and $1 \mathrm{~mm}$ in the backward and forward radiation direction, respectively. It was found that the absorbed dose delivered to the ocular globe as a result of $2 \mathrm{~mm}$ lead shielding was reduced by about $60 \%$.

\section{Acknowledgments}

This study was supported in part by Generalitat Valenciana (Project PROMETEO2008/114); and Ministerio de Ciencia e Innovación, Spain (Project No. FIS2010-17007).

\section{References}

1. Niu H, His W, Chu J, Kirk M. Dosimetric characteristics of the Leipzig surface applicators used in the high dose rate brachy radiotherapy. Med Phys 2004; 31: 3372-3377.

2. Perez-Calatayud J, Granero D, Ballester F et al. A dosimetric study of the Leipzig applicators. Int J Radiat Oncol Biol Phys 2005; 62: 579-584.

3. Hwang I, Lin S, Lin L et al. Alternative effective modality of Leipzig applicator with an electron beam for the treatment of superficial malignancies. Nuc Inst Meth A 2003; 508: 460-466.

4. Evans M, Yassa M, Podgorsak E et al. Surface applicators for high dose rate brachytherapy in aids-related Kaposi's sarcoma. Int J Radiat Oncol Biol Phys 1997; 39: 769-774.

5. Granero D, Perez-Calatayud J, Gimeno J. Design and evaluation of a HDR skin applicator with flattening filter. Med Phys 2008; 35: 495-503.

6. Musmacher J, Ghaly M, Byrnes R et al. High-dose-rate (HDR) brachytherapy with surface applicators - treatment for nonmelanomatous skin cancer. Med Phys 2006; 33: 2016.

7. Rodriguez S, Santos M, Richart J et al. High-dose-rate brachytherapy in skin cancers: Patient convenience, local control and cosmetic results. Brachytherapy 2008; 7: 159.

8. Gauden S, Egan C, Pracy M et al. HDR brachytherapy for the treatment of skin cancers using standard surface applicators. Brachytherapy 2008; 7: 159 .

9. Martinez-Monge R, Gómez-Iturriaga A. High-dose-rate brachytherapy in lower eyelid cancer. Brachytherapy 2007; 6: 227-229.

10. Perez-Calatayud J, Ballester F, Serrano M et al. Dosimetric characteristics of backscattered electrons in lead. Phys Med Biol 2000; 45: 1841-1849.

11. Das I, Chopra K. Backscatter dose perturbation in kilovoltage photon beams at high atomic number interfaces. Med Phys 1995; 22: 767-73.

12. Das I. Forward dose perturbation at high atomic number interfaces in kilovoltage x-ray beams. Med Phys 1997; 24: 1781-1787.

13. Das I, Moskvin V, Kassaee A et al. Dose perturbations at high$\mathrm{Z}$ interfaces in kilovoltage photon beams: comparison with Monte Carlo simulations and measurements. Radiation Physics and Chemistry 2002; 64: 173-179.

14. Farahani M, Eichmillerl F, McLaughlin SW. Measurement of absorbed doses near metal and dental material interfaces irradiated by $\mathrm{x}$ - and gamma-ray therapy beams. Phys Med Biol 1990; 35: 369-385.

15. Das I, Kahn F. Backscatter dose perturbation at high atomic number interfaces in megavoltage photon beams. Med Phys 1989; 16: 367-375.

16. Li X, Chu J, Chen W. Dose enhancement by a thin foil of high-Z material: a Monte Carlo study. Med Phys 1999; 26: 12451251.

17. Agostinelli S, Allison J, Amako K et al. Geant4 - A Simulation Toolkit. Nuc Ins Meth 2003; A506: 250-303. See also http:/ / geant4.web.cern.ch/geant4 last accessed 11 March 2011.
18. Yu P, Butson M, Cheung T. Does mechanical pressure on radiochromic film affect optical absorption and dosimetry? Australas Phys Eng Sci Med 2006; 29: 1-3.

19. Devic S, Seuntjens J, Sham E et al. Precise radiochromic film dosimetry using a flat-bed document scanner. Med Phys 2005; 32: 2245-2253.

20. Lynch B, Kozelka J, Ranade M et al. Important considerations for radiochromic film dosimetry with flatbed CCD scanners and EBT GAFCHROMIC film. Med Phys 2006; 33: 4551-4556.

21. Van Battum L, Hoffmans D, Piersma $\mathrm{H}$ et al. Accurate dosimetry with GafChromicTM EBT film of a $6 \mathrm{MV}$ photon beam in water: What level is achievable? Med Phys 2008; 35: 704-716.

22. Cheung T, Butson M, Yu P. Post-irradiation colouration of Grafchromic EBT radiochromic film. Phys Med Biol 2005; 50: 281-285.

23. Paelinck L, De Neve W, De Wagter C. Precautions and strategies in using a commercial flatbed scanner for radiochromic film dosimetry. Phys Med Biol 2007; 52: 231-242.

24. Richter C, Pawelke J, Karsh L et al. Energy dependence of EBT-1 radiochromic film response for photon (10 kVp-15 MVp) and electron beams (6-18 MeV) readout by a flatbed scanner. Med Phys 2009; 36: 5506-5514. 